\title{
APPROCHE DU COUPLE DEDANS-DEHORS CHEZ DELEUZE (BOGOTÁ/BARRANQUILLA, AOÛT 2005)
}

\author{
FRANÇOIS ZOURABICHVILI* \\ doi: 10.11144/Javeriana.uph37-74.acdd
}

\section{RÉSUMÉ}

Le texte que nous publions ici pour la première fois fut conçu à l'origine comme une communication pour le colloque international Deleuze: una imagen del pensamiento, événement qui a eu lieu en août 2005 à Bogotá, en Colombie.

Mots clés : Deleuze ; dedans ; dehors ; désir ; limite intérieure

Filósofo francés (1965-2006), especialista en filosofía moderna europea y contemporánea francesa, particularmente en las obras de Deleuze y Spinoza. Sus distintos trabajos -ensayos, artículos y cursos- han sido publicados en múltiples idiomas.

Para citar este artículo: Zourabichvili, F. (2020). Approche du couple dedans-dehors chez Deleuze. (Bogotá/Barranquilla, Août 2005). Universitas Philosophica, 37(74), 253-265. ISSN 0120-5323, ISSN en línea 2346-2426. doi: 10.11144/Javeriana.uph37-74.acdd. 


\title{
APPROACH TO THE COUPLE INSIDE- OUTSIDE IN DELEUZE (BOGOTA/BARRANQUILLA, AUGUST 2005)
}

\author{
ABSTRACT \\ The text that we publish here for the first time was originally conceived \\ as a presentation for the international congress Deleuze: una imagen del \\ pensamiento, held in Bogota, Colombia, in August 2005. \\ Keywords: Deleuze ; Inside ; Outside ; desire ; inner limit
}


JE RÉPONDS AUJOURD'Hui à la commande de parler du dehors et du dedans chez Deleuze. La première chose à dire c'est qu'il faudrait ne pas trop simplifier Deleuze. Certes, entre « philosophie du dedans » et «philosophie du dehors », à tout prendre, et la mort dans l'âme, je choisirai de dire avec l'Opinion, c'est une philosophie du dehors. Car, bien entendu, cette philosophie ne relativise jamais le dehors, elle ne le résorbe jamais en dernière instance, elle ne le pose pas d'abord pour l'intérioriser ensuite. Le dehors est chez Deleuze une instance ultime.

Mais, d'abord, je ne suis pas sûr que cette notion ait du sens, car « dehors », dans la langue, est un mot relatif à « dedans », et il est difficile de penser un dehors sans dedans. Il est vrai qu'à un moment donné chez Deleuze apparait un primat du dehors sur le dedans, mais c'est justement une affirmation avec laquelle, si nous essayons de le suivre, si nous voulons être philosophes comme il l'a été pour son compte, c'est-à-dire sans se payer de mots, nous devons être à la fois aventureux et prudents (comme tout aventurier d'ailleurs, sinon l'aventure tourne court).

Deuxièmement, le primat du dehors est une catégorie trop grosse, trop large en tant que telle, pour y loger Deleuze tout seul. La preuve en est que Foucault a intitulé son essai sur Blanchot «La pensée du dehors », et que le sous-titre du livre principal de Levinas, Totalité et infini, est « Essai sur l'extériorité ». Il faut donc toujours préciser par quel chemin, de quelle façon, un penseur a fait ce geste de porter le dehors au premier plan de la scène philosophique.

En outre, on trouve deux indices forts qui montrent qu'il ne faut pas mésestimer le dedans chez Deleuze : il est à la fois le philosophe de l'« extériorité des relations » et de la «différence interne »; sa conception de la mort, par exemple, consiste à dire à la fois qu'elle arrive toujours du dehors, mais qu'elle ne cesse de monter au-dedans. C'est même de là qu'il faudrait partir s'il fallait traiter complètement et rigoureusement la question.

Pour des raisons de commodité, et d'impréparation, je vais aborder la question par un biais moins abstrait : la théorie du désir. Cette théorie, en gros, s'opposait à deux choses : la théorie de la transgression et la théorie de la spontanéité.

(1) Selon la théorie de la transgression, il faut que quelque chose vous soit interdit pour que vous le désiriez, ce qui a pour conséquence que le désir a besoin d'être réprimé, et même qu'il se nourrit de sa propre répression. Sans la police, où serait le plaisir ? 
(2) Selon la théorie de la spontanéité, le désir au contraire meurt d'être réprimé, il est là tout pur, tout généreux, surabondant, explosif, débordant. Lâchons les vannes et nous n'en finirons pas de jouir et de nous exprimer.

Après Mai 68, c'était les deux Opinions dominantes : répression policière, libération sexuelle. On peut résumer de cette façon la France des années 70, sans trop caricaturer, et je dirais que les années 2000 que nous vivons actuellement ne sont pas vraiment étrangères à ce schéma d'opposition. La répression est bien plus prospère aujourd'hui qu'il y a trente ans, même s'il y a moins de gens pour le dire ; quant à la libération sexuelle, il est évident qu'elle est encore plus grande aujourd'hui qu'il y a trente ans, malgré le sida et les préservatifs (bien enten$\mathrm{du}$, cette libération est une licence plus qu'une liberté, la liberté, tout compte fait, n'ayant pas grand-chose à voir avec cela). Voilà en tout cas pourquoi, contre l'idée reçue que les années 70 seraient mortes, je les crois au contraire en train de continuer sur leur lancée.

Ce qui est mort, en revanche, même si pas complètement - et c'est notre responsabilité, ou la faute au peu de talent que nous avons -, c'est ce qui, dans les années 70 , pensait. Et ce qu'il y avait de pensée dans ces années-là pensait à la fois avec et contre le temps, exactement selon la formule par laquelle Nietzsche définissait son propre concept d'intempestif, et que Deleuze aimait bien citer : « agir sur le temps, contre le temps, en faveur - je l'espère - d'un temps à venir » (Nietzsche, 1990, cité par Deleuze, 1962, p. 122). À vrai dire, il manque dans cette phrase le « avec », qui fait toute la différence entre la pratique de Nietzsche, penseur de plus en plus isolé, et Deleuze, le Deleuze qui faisait cours dans l'ambiance qu'on sait. Il ne faisait pas cours pour une génération (cela aurait été la pire des démagogies, et certains, qui font l'amalgame, ont jugé Deleuze démagogue); il faisait cours avec et dans ce qu' il appelait lui-même, [dans Différence et répétition], un certain « air du temps » (Deleuze, 1968a, p. 1). Seulement cet air du temps était fragile, et ceux qui le respiraient le plus intensément n'avaient à leur disposition, pour l'exprimer, que des théories ou des mots d'ordre inadaptés, qui menaçaient, à l'insu de ceux-là mêmes qui les véhiculaient, d'étouffer et de faire rater ce qu'ils étaient en train de vivre (des théories comme la psychanalyse et des mots d'ordre comme le « spontanéisme » ou la « libération sexuelle » en seraient de bons exemples). 
De là L'Anti-CEdipe (Deleuze \& Guattari, 1973) et sa théorie du désir, d'après laquelle le désir, bien entendu, ne se nourrit pas de la répression - de plus en plus, la conception du manque dans la psychanalyse sera tenue pour l'alliée objective de la répression bourgeoise policière dans les affaires de mœurs. Mais le désir n'est pas non plus une force surabondante donnée d'avance, comme une source naturelle et intarissable qui coulerait spontanément de l'intérieur de chacun, et qui serait simplement empêchée de s'écouler, de se manifester au dehors. En somme, le désir n'est pas l'intériorisation d'un dehors vécu comme loi, mais il n'est pas non plus - et sur ce point l'Opinion reçue était et demeure tellement forte que Deleuze et Guattari risquaient et risquent toujours de ne pas être entendus - un mouvement de l'intérieur vers l'extérieur, une extériorisation de soi. « Extériorisez-vous! » « Ne soyez pas introvertis! » Pas de mots d'ordre plus étrangers à ce que cherche à penser L'Anti-OEdipe.

Il faut ici une remarque incidente pour éviter un malentendu. Certes, Deleuze est connu comme un philosophe de l'expression. Mais si l'on regarde ce qu'il appelle « expression », cela n'a strictement rien à voir avec un mouvement $\mathrm{du}$ dedans vers le dehors. Ce serait plutôt un mouvement d'enveloppement $d u$ dehors ${ }^{1}$. Le geste verbal le plus typique, à mes yeux, de Logique du sens (réitéré des dizaines de fois, et même dans beaucoup d'autres livres de Deleuze) est celui qui dit : « $x$ n'existe pas hors de $y$, et pourtant il differe de lui en nature $»$. Un exemple de ceci est que l'événement n'appartient pas au langage, il est extérieur au langage puisqu'il est l'attribut d'un état de choses; et pourtant il n'existe pas hors de la proposition qui le recueille et l'exprime. Un dehors enveloppé, un dehors qui n'existe pas hors de ce à quoi il est extérieur, voilà le système de l'expression selon Deleuze. À la fin de sa vie, dans l'avant-propos de Critique et clinique, Deleuze (1993) trouvera une autre manière de le dire, une manière admirable de concision. Ayant défini le critère de la création littéraire comme ce qui se passe quand un écrivain trouve des moyens de porter la langue à sa propre limite, il affirme : « La limite n'est pas en dehors du langage, elle en est le dehors... ». Et ensuite il développe : « ... elle est faite de visions et d'auditions non-langagières,

1 Voir Deleuze, Logique du sens (1969) et, bien sûr, Spinoza et le problème de l'expression (1968b). 
mais que seul le langage rend possibles » (p. 9). Le grand écrivain, l'écrivain créateur, c'est celui qui sait faire en sorte que sa langue enveloppe un dehors.

[C'est, il faut le remarquer,] la raison explicite de l'antipathie de Deleuze à l'égard de Wittgenstein - il y a une autre raison, implicite, qui est la conception mortifere que Wittgenstein se fait de la philosophie. En effet, Wittgenstein admet bien un dehors du langage (et du monde, ajoute-t-il, car « les limites de mon langage sont les limites de mon monde $\gg^{2}$ ), mais ce dehors ne peut pas être dit, il ne peut être que montré. Or, ce qu'on ne peut pas dire - selon la célèbre dernière phrase du Tractatus - cela doit être tu (et le côté religieux de Wittgenstein serait une autre différence avec Deleuze). Tandis que, pour Deleuze, c'est là justement qu'on risque enfin d'avoir quelque chose à dire, c'est là que parler sort du bavardage de l'opinion et commence à prendre de l'intérêt. D'où cette riposte splendide à la conclusion de Wittgenstein, qu'on trouve dans Qu'est-ce que la philosophie?:

Mais cette sphère du virtuel, cette Pensée-Nature, c'est ce que la logique n'est capable que de montrer, suivant un mot célèbre, sans pouvoir jamais le saisir dans des propositions, ni le rapporter à une référence. Alors la logique se tait, et elle n'est intéressante que quand elle se tait. Paradigme pour paradigme, elle rejoint alors une sorte de bouddhisme zen (1991, p. 133).

[Allons par parties] : « Mais cette sphère du virtuel », à ce moment-là, Deleuze donne au dehors de la pensée le nom de virtuel, « cette Pensée-Nature, c'est ce que la logique n'est capable que de montrer, suivant un mot célèbre, sans pouvoir jamais le saisir dans des propositions ». [Il s'agit d'un] geste d'inclusion du dehors, de mettre le dehors dedans, de faire vivre en quelque sorte en vous quelque chose qui n'est pas de vous, et dans le langage quelque chose qui n'est pas du langage. [S'il n'est donc pas possible de] « le saisir dans des propositions, ni le rapporter à une référence, [a] lors la logique se tait ». Elle se tait (1) parce que, de fait, vous ne trouverez jamais dans un traité de logique symbolique un chapitre sur des énoncés philosophiques ou littéraires, qui comportent toujours quelque chose de paradoxal; et (2) parce qu'elle se donne à elle-même, avec

2 Paraphrase de : « les frontières du langage [...] signifient les frontières de mon monde » (Wittgenstein, 1993, §5.62, p. 93). [N.D.E.] 
Wittgenstein, l'impératif de se taire. « [E]t elle n'est intéressante que quand elle se tait », c'est-à-dire : c'est là, dans ce geste d'inclure le dehors, que quelque chose comme de l'intéressant, de l'important, du remarquable survient dans la parole, contrairement aux énoncés d'une banalité affligeante sur lesquels réfléchissent les logiciens de métier. Deleuze, lui, avait une autre idée de la « logique », mot qu'il affectionnait beaucoup. « Paradigme pour paradigme, elle rejoint alors une sorte de bouddhisme zen ».

Cette remarque sur le sens du concept d'expression qui se voulait incidente, mais qui m'a emmené assez loin, nous a permis après tout de croiser cette figure du dehors enveloppé, du dehors dedans, et ainsi à compliquer cette affaire du dehors chez Deleuze, selon mon intention initiale.

Je retourne donc au désir. Si le désir n'est pas ex-pressif, cette fois au sens vulgaire du terme (mouvement du dedans vers le dehors), cela veut dire que le désir n'est jamais là d'avance : nous ne savons pas d'avance quels sont nos désirs, et si nous croyons que cette ignorance tient seulement à ce qu'ils sont réprimés, nous nous préparons la plus grande des désillusions lorsque les interdits auront été levés. Tel est le sort de tous ceux qui se sont laissé berner par ladite « libération sexuelle » qui, historiquement, aura coïncidé avec une gigantesque panne de désir planétaire (du moins, soyons décents, dans cette petite partie du monde privilégiée où cette levée des interdits a pu se produire). On a cru que le désir coulerait enfin de lui-même, lui qui était comme un forcené retenu de force dans sa camisole morale et policière. On s'est retrouvé avec un désir riquiqui, vite écœuré, malgré l'étendue des possibles qui s'ouvrait soudain à lui (à tous les niveaux).

Deleuze n'aimait pas le possible. Je me permets de partager le souvenir d'un cours où, tout à coup, au bout d'une heure peut-être de cheminement patient, les concepts et les énoncés s'étaient mis à s'enchaîner pour ainsi dire tous seuls, et l'œil de Deleuze brillait, et voici ce qu'il avait dit (ou du moins ce qui m'en reste d'après les notes que j'avais prises) : « ça fait partie des moments heureux, en philosophie, quand on n'a plus le choix ». C'est surprenant, à 20 ans, quand on entend une chose pareille. On ne la comprend pas vraiment, mais on sent déjà qu'on ne l'oubliera jamais, c'est comme une merveille un peu effrayante dont on ne sait pas quoi faire.

Pourquoi est-ce heureux de ne plus avoir le choix ? Pourquoi avoir le choix est-il toujours si triste ? La réponse est toujours dans cette théorie du désir de 
L'Anti-CEdipe, que je simplifie beaucoup afin de mettre en relief sa manière originale et importante de s'orienter en fonction des valeurs du dehors et du dedans. Deleuze et Guattari croient tellement peu à l'intériorité que la répression du désir relève pour eux d'un faux problème. Ou plutôt d'un problème mal posé. II y a une répression du désir, mais elle n'est pas celle qu'on croit : c'est la répression qui porte sur la production du désir. C'est le discours qui consiste à nous faire croire : ton désir, tu l'as à l'avance, il est déterminé d'avance.

On retrouve ici les deux options dont j'avais parlé avant, transgression et spontanéité : (1) il est déterminé à l'avance par les interdits auxquels tu te confrontes (donc, bénis-les, sans quoi tu n'aurais pas de désir, mais préfere quand même ne pas transgresser, car le désir est chose infantile, il faut savoir grandir) ; (2) il est déterminé à l'avance parce que chacun a sa richesse propre, son originalité propre, sa personnalité, son moi, son identité (tout le monde sait qu'une identité conduit à des ancêtres et a des racines, mais on ne s'embarrasse pas de la contradiction et on croit quand même que c'est ce qui nous est le plus propre). En bref, tu es d'avance propre, tes propriétés te sont innées, tu es d'avance toi-même, et tu n'as qu'à t'exprimer (c'est là, soit dit en passant, que Deleuze parlait de l'horreur de la situation où l'on vous dit « Camarade, exprime-toi $!$ », et il disait toujours qu'il n'avait rien à dire, rien de spécial à exprimer - parler, c'était pour lui toute autre chose que s'exprimer, au sens de faire surgir ce qu'on a au fond de soi).

[Pour résumer], ce sont deux discours qui reviennent au même, dans la mesure où tous deux ont pour présupposé que le désir est déterminé d'avance. Or ouvrons les vannes, supprimons les entraves, levons les interdits : la seule révélation prévisible au bout de quelque temps est que le roi est nu. À savoir : le désir est quelque chose qui se construit, la répression ne portait pas comme on le croyait sur des désirs prêts à s'exprimer, mais sur la production du désir, elle consistait à nous masquer que le désir est d'abord productivité, processus de production.

Or si le désir n'est déterminé à l'avance ni par le moi ni par la loi, ni par un dedans naturellement riche ni par un dehors artificiellement imposé, d'où vientil ? C'est là que Deleuze parle du «plan d'immanence » comme du «Dehors d'où vient tout désir » (Deleuze \& Parnet, 1996, p. 116 ; mes soulignés). Cette expression doit se prendre au moins à deux niveaux.

À un premier niveau, le désir a pour condition une rencontre, la relation avec quelque chose qui n'est pas soi. Question difficile, parce que ce concept est 
construit à partir d'un motif qui a l'air très simple, qui a même l'air un peu trop simple, et qui vient de Spinoza. Chacun - individu, chose, etc. - se définit par un ensemble de rapports, et la question est de savoir si deux individus arriveront à composer leurs rapports ou bien s'autodétruiront, y compris en ayant l'impression de (se) composer. C'est peut-être d'une certaine vérité, mais c'est une vérité comme telle un peu banale. En réalité, ce motif de la composition de rapports prend tout son sens (et donc son intérêt) au niveau du concept d' « haeccéité », c'est-à-dire, à propos de comment des choses très différentes en nature, indépendamment de leurs différences de règnes, de genre et d'espèces, arrivent à se composer entre elles, au niveau de purs rapports de vitesse. Mais nous ne ferons pas ce trajet aujourd'hui.

Voici les trois cas qui ne sont pas intéressants :

- soit on se ressemble, donc pas de problème de composition, on s'entend d'avance et on risque de s'ennuyer une fois qu'on a reconnu tout ce qu'on avait en commun et qu'on est d'accord sur tout ;

- soit on est incompatible et c'est destructeur, qu'il s'agisse d'aliments, de musique ou de personnes;

- soit, troisièmement, on est différent, mais ces différences ne nous font rien, n'ont rien d'attirant pour soi, ne déclenchent aucune émotion (contre le mot d'ordre de ces 20 dernières années selon lequel nos différences nous enrichissent - d'abord il ne s'agit jamais dans la vie de s'enrichir, ensuite il y a des différences qui ne nous font rien, qui ne nous touchent pas).

Donc le problème de Deleuze et Guattari était de comprendre l'imprévisible de la rencontre, à savoir : comment se peut-il que nous soyons attirés par une différence. Encore une fois, il n'y a rien de mécanique dans le fait d'être confronté à la différence : parfois ça marche et quelque chose se passe, parfois rien ne se passe, et seule la nouvelle morale de la différence est là pour nous culpabiliser et nous suggérer que nous sommes en train de négliger une minorité et donc potentiellement d'en faire une victime. Deleuze réclamait le droit de ne pas s'intéresser à tout, il disait : «j'aime peu de choses », mais - ajoute-t-on - ce que j'aime, je vis avec, ça m'est absolument nécessaire.

Or, ce qu'il aime ou ce que chacun aime, que sont toutes ces pentes d'émotion en nous ? Comme souvent en philosophie, la réponse est dans la question : c'est ce qui nous touche. Deleuze et Guattari vont appeler cela des « lignes de 
fuite », des « pointes de déterritorialisation ». Et ici je suis obligé d'aller vite. D'abord, il ne faut pas croire que ce que nous rencontrons agit sur nous comme une force de déterritorialisation brute. Ce serait tout simplement insupportable, mortel. Violence pure. Mais il y a une violence dans toute rencontre au sens fort (entendons : la rencontre où il se passe quelque chose, pas la visite d'un lieu ou d'un musée qui ne vous dit rien, ou la rencontre de personnes avec lesquelles vous n'avez que la ressource d'être poli et si possible divertissant jusqu'à ce que la soirée se termine). Cette violence peut être terrible, changer complètement notre vie ou notre perspective sur la vie. Mais cette violence neutre n'a pu s'exercer que parce qu'elle touchait et nous faisait toucher nos propres points sensibles, points d'émotions, points de joie et d'angoisse. Elle nous mettait brusquement en rapport avec nous-mêmes. Et elle le faisait dans des conditions qui n'étaient pas du tout cognitives. Il ne s'agit pas du tout de dire qu'une rencontre aide à mieux nous connaître (ce serait le même genre de banalité que l'enrichissement mécanique des différences les unes par les autres). Car s'affecter soi-même (1) n'est pas un rapport de nature théorique, ou cognitive, mais un rapport de désir ; la sensibilité ayant affaire à elle-même ne se connaît pas, mais se sent, et sent quelque chose qu'elle n'avait pas encore senti, ou plus exactement comme elle ne l'avait jamais senti ; (2) n'est pas une relation intérieure, du type de la réflexivité qui, à l'âge classique, constitue « l'intériorité » : un rapport immédiat de soi à soi, par une propriété spontanée de l'esprit qui est d'être conscient de soi.

Pour toucher à soi-même comme on ne s'est jamais touché, comme on ne s'est jamais senti, il n'y a pas ici la généralité d'une expérience introspective qu'on pourrait mener seul, comme dans la phénoménologie. Il faut la rencontre la plus imprévisible et la plus incongrue, celle qui à la fois sait nous atteindre au tréfonds, et nous atteindre avec une perspective que nous n'avions pas, que nous n'aurions pas su inventer, qui [...] n'était pas possible, n'était pas dans l'ordre des possibilités disponibles, des sensations reconnaissables, des vécus anticipables. Et c'est pourquoi la rencontre instaure simultanément un territoire et nous déplace, nous attire, nous entraîne sur des lignes déterritorialisantes. Elle transforme en devenirs ce qui n'était que points d'angoisse conjurés. Nous ne nous rapportons à nous-mêmes qu'en devenir, car se rapporter à soi-même est affaire de désir et non de connaissance. Et nous ne nous atteignons nous-mêmes que sous l'action de quelque chose qui nous déplace. Ou seul ce qui nous déplace est 
à même de nous rapporter à nous-mêmes - toujours dans un circuit ou la familiarité et l'étrangeté s'enchaînent et se mêlent l'une à l'autre. Nous ne visitons ce qui nous hante, nous ne sommes sur les bords remodelables de nous-mêmes, qu’à la condition d'une rencontre.

Si j'allais plus loin, j'arriverais au concept de « ritournelle ». Mais ce n'est pas nécessaire, car il suffit d'éclaircir un peu mieux cette situation du désir par rapport au couple dedans-dehors. En effet, la formule « le Dehors d'où vient tout désir » a deux sens. Le premier sens est que la condition pour que le désir - qui ne coule jamais d'une source naturelle enfouie au plus intime de nous-mêmes - trouve à couler, c'est justement une rencontre, donc un agent extérieur. Le deuxième sens est indiqué par Deleuze dans le texte même d'où j'extrais cette formule : ce dehors, c'est le «plan d'immanence ». Autrement dit, le plan même où l'expérience - la rencontre - a lieu. La rencontre s'effectue physiquement : vous tombez amoureux, ou le petit Hans voit un cheval tirant un omnibus s'effondrer dans la rue et battre des jambes dans tous les sens sous les coups de fouet, ou c'est la rencontre d'une musique, d'un livre, d'un lieu, d'un groupe, ou de toute autre chose encore. Là, le dehors est ce qui est matériellement distinct de vous, ce qui est venu à vous d'une manière ou d'une autre. Mais si vous dites que ça vous est tombé dessus, que ça agit maintenant comme une vrille au-dedans de vous, ce n'est plus un processus spatialisable. Cela se passe d'une certaine manière «dedans », c'est intime. Le dehors, en tant qu'il vous affecte, est intime. Il se définit comme l'irruption dans votre pensée d'une autre manière de penser, dans votre sensibilité d'une autre manière de sentir. Et pourtant cette intimité n'est rien de connu ou de reconnaissable par vous. Elle est votre seule vraie intimité (1'autre c'est celle de l'adolescent qui se creuse la tête en essayant de se confesser dans son journal intime et qui ne trouve que des platitudes à dire, précisément parce qu'il croit devoir chercher en lui-même, faire l'inspection de son moi le plus pur, le plus personnel, en faisant abstraction de tout dehors). «Le Dehors d'où vient tout désir », par conséquent, c'est l'intime en tant qu'il se construit en s'échappant à lui-même sous la violence de ce qui le touche définitivement. C'est le plan d'immanence, et ce plan est un territoire, c'est-à-dire ce à quoi nous revenons sans arrêt comme à ce qui nous concerne vraiment, une sorte d'apprivoisement du terrible à partir de la perspective ouverte par la rencontre. 
Le dehors est donc cette limite intérieure dont l'expérience a pour condition la rencontre, dans sa double dimension d'irruption et de composition. Il y a composition - on le comprend maintenant - pour autant que la perspective inédite nous arrache à nous-mêmes (à nos régularités, à nos rengaines, aux généralités à travers lesquelles nous identifions notre vie et nous reconnaissons nousmêmes) pour faire de nos failles, de nos points sensibles ou de nos hantises des devenirs (plutôt que des îlots angoissants que nous ne visitons jamais).

C'est pourquoi le dehors, pour Deleuze, n'est pas seulement au dehors, par opposition à un dedans avec lequel il y aurait une frontière étanche. Au contraire, les milieux dans lesquels nous vivons, le temps qu'il fait et qui change, la chaleur ou le froid, les gens que nous fréquentons, les scènes furtives auxquelles nous assistons, les livres que nous lisons - tout cela évidemment ne nous reste pas extérieur, mais travaille en nous. Le problème, c'est que si nous disons, « moi c'est moi et les autres c'est les autres », « moi c'est moi et le dehors est le dehors », si nous vivons comme ça, si à un moment donné le ciel qui change ce n'est pas moi, le cheval qui tombe [n'est pas] le petit Hans, ou cette musique comme un morceau de moi-même que je ne peux retrouver et retoucher qu'en la réécoutant, ne sont pas moi, alors le dehors ne s'enveloppe pas, alors le désir ne se construit pas, et il ne reste qu'une intimité creuse. Et cette intimité creuse est toute constituée en réalité d'extériorité factice, c'est-à-dire de catégories générales qui permettent de répartir le possible : j'ai tel métier plutôt que tel autre, je suis marié plutôt que célibataire, j'aime le café plutôt que le chocolat, l'Asie plutôt que l'Afrique - en bref, je ne suis qu'une série de propriétés générales. Chez Deleuze, le dehors n'est donc pas tout ce qui n'est pas moi (un ensemble déterminable matériellement), mais tout ce qui me sort de moi, la limite de ma pensée ou de ma sensibilité pour autant que cette limite elle-même peut me devenir sensible, c'est-à-dire pour autant que je sais envelopper un dehors.

Pour conclure, [je dirai ceci] : aucun grand philosophe, que je sache, n'a cru à l'intériorité pleine, naturellement riche. L'intériorité du sage stoïcien, par exemple, est vide, et justement il faut qu'elle se concentre sur sa vacuité, sur la pure forme du « ce qui dépend de moi », la volonté d'adhérer à ce qui arrive, quoi qu'il arrive. L'intériorité pleine, on ne la trouve peut-être que chez Hegel, comme la fin même d'un processus infini d'intériorisation (l'intériorisation n'est pas du tout l'enveloppement du dehors comme tel : elle annule l'extériorité, 
elle signifie son assimilation). Pour le reste, et notamment dans la philosophie chrétienne, l'intériorité est toujours en dernière instance en prise sur un dehors : saint Augustin dit bien que Dieu m'est plus intérieur encore que mon intimité même, ce qui veut dire aussi réciproquement que Dieu est un dehors plus lointain que le monde extérieur. Et tout l'âge classique chrétien, Descartes compris, reprend ce motif. On trouve ce motif explicitement chez Deleuze, dans L'imagetemps (1985), mais la grande différence est que pour lui le dehors, si radical soitil, est immanent. Autrement dit, les rencontres ne proviennent que du monde. Immanence ne veut plus dire alors : l'être humain laissé à sa finitude et sans perspective de rupture (« rien de nouveau sous le soleil »). Le monde porte en lui la différence, le dehors, le déplacement des perspectives, c’est pourquoi il est essentiellement virtuel, irréductible à un champ de possibilités ouvert pour toujours. C'est la dernière manière dont le dehors s'enveloppe, chez Deleuze, sans qu'il $\mathrm{y}$ ait pour autant retournement du primat du dehors en primat du dedans : le dehors est dans le monde. Et c'est pourquoi - un des cris philosophiques ultimes de Deleuze - il faut réapprendre à croire au monde.

\section{Références}

Deleuze, G. (1962). Nietzsche et la philosophie. Paris : PUF.

Deleuze, G. (1968a). Différence et répétition. Paris : PUF.

Deleuze, G. (1968b). Spinoza et le problème de l'expression. Paris : Les Éditions de Minuit.

Deleuze, G. (1969). Logique du sens. Paris : Les Éditions de Minuit.

Deleuze, G. (1985). L'image-temps. Cinéma 2. Paris : Les Éditions de Minuit.

Deleuze, G. (1991). Qu'est-ce que la philosophie? Paris : Les Éditions de Minuit.

Deleuze, G. (1993). Critique et clinique. Paris : Les Éditions de Minuit.

Deleuze, G. \& Guattari, F. (1973). L'Anti-OEdipe. Capitalisme et schizophrénie. Paris : Les Éditions de Minuit.

Deleuze, G. \& Parnet, C. (1996). Dialogues. Paris : Flammarion.

Nietzsche, F. (1990). Considérations inactuelles I et II. (Ed. G. Colli \& M. Montinari ; Trad. P. Rusch). Paris : Gallimard.

Wittgenstein, L. (1993). Tractatus logico-philosophicus. (Trad. G. G. Granger). Paris : Gallimard. 